\title{
Prevalencia de ansiedad en estudiantes universitarios ${ }^{*}$
}

\section{Prevalence of anxiety in students of a university}

\author{
Jaiberth A. Cardona-Arias** \\ Universidad Cooperativa de Colombia \\ Universidad de Antioquia, Colombia \\ Diana Pérez-Restrepo \\ Stefanía Rivera-Ocampo \\ Jessica Gómez-Martínez \\ Ángela Reyes \\ Universidad Cooperativa de Colombia \\ Recibido: 12 de septiembre 2014 \\ Revisado: 23 de octubre de 2014 \\ Aceptado: 18 de diciembre de 2014
}

\section{Resumen}

Los universitarios constituyen un grupo de riesgo para la ansiedad por sus exigencias psicológicas, sociales, académicas y elevado estrés. El objetivo de este trabajo fue determinar la prevalencia de ansiedad en estudiantes de una universidad privada de Medellín y su asociación con aspectos sociodemográficos y académicos. El método de estudio de prevalencia se llevó a cabo con 200 estudiantes seleccionados mediante muestreo probabilístico estratificado. La tamización de ansiedad se realizó con la escala de Zung. Se evaluó la fiabilidad de la escala con el a de Cronbach, el análisis se realizó con medidas de resumen, frecuencias, $U$ de Mann Whitney y chi cuadrado, en SPSS $21 \circledast$. Los resultados evidencian que la prevalencia de ansiedad fue $58 \%$. La escala de Zung presentó una excelente fiabilidad con a de Cronbach de 0,8. La prevalencia de ansiedad presentó una distribución estadísticamente igual según el sexo y el grupo etario; no se halló asociación con el ciclo de formación, el programa académico, la edad, los créditos matriculados, ni la ocupación. La ansiedad fue estadísticamente menor en los estudiantes de clase media. Se halló una elevada prevalencia de ansiedad, lo que pone de manifiesto la

Artículo de investigación.

Correspondencia: Jaiberth Antonio Cardona Arias. Calle 67 \# 53-108, bloque 5, oficina 103, Medellín, Colombia. Teléfono: 2198486. Fax: 2195486. Correo electrónico jaiberthcardona@gmail.com 
necesidad de desarrollar de estrategias educativas, identificar estudiantes de mayor riesgo e iniciar intervenciones en salud de manera oportuna.

Palabras clave: prevalencia, ansiedad, estudiantes, medicina, Colombia.

\section{Abstract}

College students are a risk group for anxiety for their psychological, social and academic demands, and high stress. In order to determine the prevalence of anxiety in students from a private university in Medellin and its association with socio-demographic and academics, a prevalence study with 200 students selected using stratified probability sampling was carried out. The anxiety screening was performed with the Zung scale. We assessed the reliability of the scale with Cronbach's $\alpha$, the analysis was performed using summary measures, frequencies, Mann Whitney's $U$ and chi square in SPSS $21 \AA$. The prevalence of anxiety was $58 \%$. The Zung scale showed excellent reliability with Cronbach's a of 0.8 . The prevalence of anxiety presented a statistically equal distribution by sex and age group, no association was found with the training cycle, academic program, age, credits or occupation. Anxiety was statistically lower in middle-class students. We found a high prevalence of anxiety, which highlights the need to develop educational strategies, identify high-risk students and initiate health interventions opportunely.

Keywords: Prevalence, anxiety, students, medicine, Colombia.

\section{Introducción}

La época universitaria constituye un periodo de la vida donde la mayoría de los estudiantes son adolescentes o adultos jóvenes, lo que implica que son una población donde se consolidan los proyectos de vida, se adquiere la mayoría de edad, se asumen nuevas responsabilidades sociales y aumentan las presiones psicosociales, lo que puede redundar en una mayor vulnerabilidad frente a trastornos psicosociales como el síndrome ansioso.

Todos los individuos experimentan ansiedad en alguna etapa de su vida; esta se caracteriza por una sensación de aprensión difusa, desagradable y vaga, que se manifiesta por la incapacidad de estar sentado o de pie durante un tiempo, y a menudo se acompaña de síntomas vegetativos como cefalea, diaforesis, taquicardia, opresión torácica, malestar epigástrico e inquietud. En una condición clínica normal, es una señal de alerta que advierte de un peligro inminente y permite al individuo tomar medidas para afrontar dicha amenaza (Kaplan, Sandock y Sadock, 2009).
La ansiedad puede presentarse en tres formas: 1) normal, en la cual hay manifestaciones afectivas como respuesta a un estímulo tanto del mundo exterior como interior; 2) patológica, donde, a diferencia de la normal, no hay un estímulo previo; y 3) la generalizada, que se caracteriza por miedos prolongados vagos e inexplicables sin relación con un objeto (Acuña, Botto y Jiménez, 2009; Sarason y Sarason, 1996).

En Colombia se presentan múltiples situaciones que aumentan el riesgo de padecer trastornos en la salud mental, particularmente ansiedad, las cuales incluyen dificultades en el acceso a educación, intensa migración urbana, falta de capacitación laboral, delincuencia y violencia juveniles, consumo de sustancias tóxicas y embarazo no deseado (Alba, 2010).

Según la Encuesta Nacional de Salud Mental de 2003 de Colombia, la prevalencia de vida de cualquier trastorno mental en la población general fue de $40,1 \%$, siendo los adolescentes y adultos jóvenes los más afectados; entre dichos trastor- 
nos, los más altos correspondieron a ansiedad [las edades promedio de inicio de fobia social, agorafobia y trastorno de pánico fueron 14,16 y 18 años, respectivamente (Alba, 2010)].

En Colombia, los trastornos de ansiedad son de mayor prevalencia que otros trastornos mentales; el 19,3\% de la población entre 18 y 65 años la ha padecido alguna vez en la vida (en mujeres, la prevalencia aumenta a 21,8\%). La región del Pacífico y Bogotá son las que presentan la prevalencia más elevada de trastorno de ansiedad, con $22,0 \%$ y $21,7 \%$, respectivamente, mientras que la región Atlántica presenta la menor, con 14,9\%. Estudios previos han reportado que solo el $1,9 \%$ de los pacientes acude al psiquiatra; el 5,6\%, al médico general; y el 3,9\% optan por tratamientos de la medicina alternativa (Posada, Buitrago, Medina y Rodríguez, 2006; Ministerio de la Protección Social de Colombia, 2003; Posada, Aguilar y Gómez, 2004).

La frecuencia de trastornos mentales en general, y la ansiedad en particular, es mayor en poblaciones con factores de riesgo sociales, clínicos y ambientales, los cuales presentan una frecuencia diferente según la población estudiada, aspecto que resalta la necesidad de desarrollar investigaciones en poblaciones específicas. Concretamente, los estudiantes universitarios presentan con mayor frecuencia este tipo de riesgos, y constituyen uno de los grupos de mayor interés para el estudio de la ansiedad, debido a sus exigencias psicológicas, sociales y académicas, aunadas a los altos niveles de estrés que, en conjunto, conllevan a problemas de salud mental como trastornos depresivos, ansiedad, suicidio, abuso de alcohol y drogas, entre otros (Bohórquez, 2007).

A pesar de la importancia de estudiar la ansiedad en estudiantes universitarios y de disponer de un instrumento validado para su tamización, como el test de Zung, en la Universidad Cooperativa de Colombia, sede Medellín, no se han realizado estudios sobre su prevalencia y factores asociados.

Unida al desconocimiento de este tópico, la importancia de su estudio radica en que es un problema que afecta la salud física, emocional y social, lo cual se agrava en personas que están con presiones laborales o académicas, como sucede en la educación superior universitaria. Además, las investigaciones en este tema permitirían formular métodos de estudio y estrategias que, a futuro, ayuden a identificar los factores de riesgo que llevan a una complicación, establecer o desarrollar un plan de detección e intervención de casos, y, en general, evitar las repercusiones negativas de la ansiedad en el desempeño académico y la calidad de vida.

En coherencia con lo expuesto, se realizó un estudio con el objetivo de determinar la prevalencia de ansiedad en estudiantes de una universidad privada de Medellín y su asociación con aspectos sociodemográficos y académicos.

\section{Materiales y métodos}

\section{Tipo de estudio: de prevalencia.}

Sujetos de estudio: 100 estudiantes de ingeniería y 100 de medicina de la Universidad Cooperativa de Colombia, sede en Medellín. El tamaño de la muestra correspondió a una población de referencia de 1050 estudiantes de una sede de la Universidad Cooperativa, donde existe un programa de medicina y 5 de ingeniería, una proporción esperada de ansiedad de $20 \%$, confianza de $95 \%$ y error de muestreo de $5 \%$. Para la selección de los estudiantes se realizó un muestreo probabilístico estratificado, con asignación proporcional según el programa; este se agrupó en medicina y otros.

Criterios de inclusión: 1) personas con matrícula vigente, 2) individuos de ambos sexos, 3) sujetos de cualquier edad. Criterios de exclusión: 3 ) personas con tratamientos ansiolíticos, 2) consumidores de alucinógenos, 3) exigir remuneración por participar del estudio, 4) no firmar el consentimiento informado.

Recolección de la información: se empleó información primaria, consistente en una encuesta estructurada con preguntas dicótomas y politómicas, con aplicación individual, anónima y asistida. Previo a la recolección de la información, los participantes firmaron un consentimiento infor- 
mado. Para el control de sesgos de información en los encuestadores, se estandarizó el diligenciamiento de la información y se realizó un manual de trabajo de campo con protocolo de llegada, presentación, ejecución y despedida; se motivó la participación de los encuestados a través de la información del proyecto dada por los encuestadores, los investigadores y la consignada en el consentimiento informado, una carta de presentación institucional con los números de teléfono y ubicación de los investigadores, y se garantizó la confidencialidad en la información recolectada. En el instrumento se realizó una prueba piloto y se redactó un formulario de manera lógica, clara y coherente, sin condicionar las respuestas de los participantes, y se realizó doble digitación de la información.

Tamización de ansiedad: se realizó a través de la escala de Zung validada para Colombia; esta presenta 20 puntos -15 relacionados con aspectos negativos como nerviosismo, temor, alteraciones del sueño o pánico, temblor, dolor de cabeza, debilidad y cansancio, taquicardia, mareos, desmayos, adormecimiento o parestesias, indigestión, poliuria, bochornos y pesadillas, y 5 relacionados con aspectos positivos como bienestar, tranquilidad, respiración normal, temperatura de manos normal y facilidad para dormir-. Los primeros se clasifican en una escala de 1 a 4, donde 1 es nunca (mejor resultado) y 4 es siempre (peor resultado), mientras que en los segundos se invierte la escala Likert ( 1 es siempre y 4 nunca). Con ello se obtiene un puntaje entre 20 y 80 , el cual se multiplica por 1,25 para generar un resultado global entre 25 y 100 , donde los puntajes más elevados indican mayor grado o riesgo de ansiedad. En esta escala los individuos con 45 o más puntos presentan ansiedad; además, se genera una variable ordinal categorizada de la siguiente manera: 1) sin ansiedad, entre 24-44; 2 ) ansiedad leve, entre 45-64; 3) ansiedad moderada, entre 65-74; y 4) ansiedad grave, entre 75-100 (Bohórquez, 2007).
Análisis de la información: para la descripción de la población de estudio y la determinación de la prevalencia de ansiedad se estimaron proporciones y medidas de resumen. Para describir el puntaje obtenido con el test de Zung se emplearon medidas de resumen, y para analizar su fiabilidad, se calculó el $a$ de Cronbach y se determinó la significación estadística del coeficiente de correlación de cada punto de la escala con el puntaje global. Para comparar la prevalencia de ansiedad según sexo, grupo etario, estrato social, ciclo de formación, programa académico y ocupación se realizó la prueba chi cuadrado de Pearson, y según la edad y el número de créditos matriculados, la U de Mann-Whitney, dado el incumplimiento del supuesto de normalidad evaluado con la prueba de Kolmogorov Smirnov con corrección de la significación de Lilliefors. Los datos se almacenaron en una base de datos en SPSS versión $20,1 \circledR$. En todos los análisis se tomó una significación estadística de 0,05 .

Aspectos éticos: En todas las etapas del proyecto se tuvieron presentes los principios de la declaración de Helsinki y la resolución 8430 de Colombia, según la cual el estudio corresponde a una investigación sin riesgo. Prevaleció el respeto a la dignidad de los sujetos y la protección de sus derechos y de su privacidad. El proyecto contó con aval del Comité de Bioética de la Universidad Cooperativa de Colombia.

\section{Resultados}

La edad presentó una media de 22 años; el $50 \%$ de los valores centrales estuvo entre 20 y 23 , y el rango entre 17 y 39 años. En los créditos matriculados el promedio fue 18, con rango entre 6 y 23. El 53\% fueron hombres; el 34,5\%, adolescentes; y $66,5 \%$, clase media. La prevalencia de ansiedad fue de $58 \%$, con un $55 \%$ en grado leve (tabla 1 , al final del artículo). 
Tabla 1

Descripción del grupo de estudio y prevalencia de ansiedad

\begin{tabular}{|c|c|c|c|}
\hline & & $\mathrm{X} \pm \mathrm{DE}$ & $\operatorname{Me}(\mathrm{RI})$ \\
\hline Edad (en años cumplidos) & & $22,0 \pm 3,2$ & $22(20-23)$ \\
\hline \multirow[t]{2}{*}{ Créditos matriculados } & & $17,9 \pm 4,6$ & $20(15-22)$ \\
\hline & & $\#$ & $\%$ \\
\hline \multirow{2}{*}{ sexo } & Masculino & 106 & 53,0 \\
\hline & Femenino & 94 & 47,0 \\
\hline \multirow{2}{*}{ Grupo etario } & Adolescente & 69 & 34,5 \\
\hline & Adulto medio & 131 & 65,5 \\
\hline \multirow{3}{*}{ Estrato social } & Bajo (1-2) & 20 & 10,0 \\
\hline & Medio (3-4) & 133 & 66,5 \\
\hline & Alto $(5-6)$ & 47 & 23,5 \\
\hline \multirow{3}{*}{ Ciclo de formación } & Básico (1-4) & 73 & 36,5 \\
\hline & Profesional (5-8) & 92 & 46,0 \\
\hline & Profundización (>8) & 35 & 17,5 \\
\hline \multirow{2}{*}{ Programa } & Medicina & 100 & 50,0 \\
\hline & Otros & 100 & 50,0 \\
\hline \multirow{2}{*}{ Ocupación } & Estudia & 187 & 93,5 \\
\hline & Estudia y trabaja & 13 & 6,5 \\
\hline \multirow{4}{*}{ Prevalencia de ansiedad } & Sin ansiedad & 84 & 42,0 \\
\hline & Con ansiedad (cualquier grado) & 116 & 58,0 \\
\hline & Ansiedad leve & 111 & 55,5 \\
\hline & Ansiedad moderada & 5 & 2,5 \\
\hline
\end{tabular}

X: Media. DE: Desviación Estándar. Me: Mediana. RI: Rango Intecuartil.

Fuente: elaboración propia. 
El puntaje obtenido con el test se Zung fluctuó entre 29 y 74 puntos, con una media de 47 puntos; la escala presentó una excelente fiabilidad en el grupo de estudio con $a$ de Cronbach de 0,8 . En los puntos que conforman esta escala, los que pre- sentaron la mayor correlación con el puntaje final fueron los relacionados con temor, tranquilidad y taquicardia; por su parte, los de menor correlación fueron la percepción de bienestar, dolor y sensación de manos secas y calientes (tabla 2).

Tabla 2

Análisis de fiabilidad y descripción del test de Zung

\begin{tabular}{|c|c|c|c|c|c|}
\hline \multirow{2}{*}{ Punto o ítem evaluado } & \multicolumn{4}{|c|}{ Frecuencia referida (\%) } & \multirow{2}{*}{$\begin{array}{l}\text { Correlación con } \\
\text { puntaje global }\end{array}$} \\
\hline & Nunca & A veces & Con frecuencia & Siempre & \\
\hline Nerviosismo & $88(44,0)$ & $84(42,0)$ & $20(10,0)$ & $8(4,0)$ & 0,390 \\
\hline Temor & $119(59,5)$ & $67(33,5)$ & $11(5,5)$ & $3(1,5)$ & 0,538 \\
\hline Pánico & $102(51,0)$ & $70(35,0)$ & $22(11,0)$ & $6(3,0)$ & 0,462 \\
\hline "Me siento reventar" & $128(64,0)$ & $51(25,5)$ & $17(8,5)$ & $4(2,0)$ & 0,494 \\
\hline Bienestar & $38(19,0)$ & $76(38,0)$ & $50(25,0)$ & $36(18,0)$ & 0,225 \\
\hline Temblor & $96(48,0)$ & $81(40,5)$ & $14(7,0)$ & $9(4,5)$ & 0,419 \\
\hline Dolores & $82(41,0)$ & $76(38,0)$ & $34(17,0)$ & $8(4,0)$ & 0,281 \\
\hline Debilidad-cansancio & $83(41,5)$ & $79(39,5)$ & $30(15,0)$ & $8(4,0)$ & 0,442 \\
\hline Tranquilidad & $45(22,5)$ & $55(27,5)$ & $49(24,5)$ & $51(25,5)$ & 0,521 \\
\hline Taquicardia & $83(41,5)$ & $82(41,0)$ & $24(12,0)$ & $11(5,5)$ & 0,511 \\
\hline Mareo & $136(68,0)$ & $41(20,5)$ & $18(9,0)$ & $5(2,5)$ & 0,442 \\
\hline Desmayo & $152(76,0)$ & $28(14,0)$ & $13(6,5)$ & $7(3,5)$ & 0,486 \\
\hline Respiración normal & $42(21,0)$ & $30(15,0)$ & $30(15,0)$ & $97(48,5)$ & 0,427 \\
\hline Parestesia & $137(68,5)$ & $41(20,5)$ & $12(6,0)$ & $9(4,5)$ & 0,458 \\
\hline Indigestión & $84(42,0)$ & $71(35,5)$ & $26(13,0)$ & $19(9,5)$ & 0,340 \\
\hline Poliuria & $74(37,0)$ & $70(35,0)$ & $44(22,0)$ & $12(6,0)$ & 0,215 \\
\hline Manos secas & $88(44,0)$ & $60(30,0)$ & $18(9,0)$ & $34(17,0)$ & 0,124 \\
\hline Bochornos & $91(45,5)$ & $73(36,5)$ & $23(11,5)$ & $13(6,5)$ & 0,366 \\
\hline Descanso & $40(20,0)$ & $51(25,5)$ & $40(20,0)$ & $68(34,0)$ & 0,292 \\
\hline \multirow[t]{2}{*}{ Pesadillas } & $87(43,5)$ & $69(34,5)$ & $29(14,5)$ & $15(7,5)$ & 0,404 \\
\hline & Media \pm D.E. & Mediana & RI & Rango & a Cronbach \\
\hline Puntaje global & $47,28 \pm 8,594$ & 46,25 & $41,3-53,8$ & $29-74$ & 0,771 \\
\hline
\end{tabular}

DE: Desviación Estándar. RI: Rango Intercuartil.

Fuente: elaboración propia. 
La prevalencia de ansiedad presentó una distribución estadísticamente igual según el sexo y el grupo etario; además no presentó asociación con el ciclo de formación, el programa académico ni la ocupación. Dicha prevalencia solo presentó una asociación estadísticamente significativa con el estrato social, siendo menor en los estudiantes de clase media (tabla 3).

Tabla 3

Comparación de la prevalencia de ansiedad según condiciones sociodemográficas y académicas

\begin{tabular}{|c|c|c|c|c|c|c|}
\hline & & \multicolumn{4}{|c|}{ Ansiedad } & \multirow{3}{*}{ Valor $\mathrm{p} \mathrm{Chi}{ }^{2}$} \\
\hline & & \multicolumn{2}{|c|}{ Normal } & \multicolumn{2}{|c|}{ Ansiedad } & \\
\hline & & \# & $\%$ & \# & $\%$ & \\
\hline \multirow{2}{*}{ Sexo } & Masculino & 46 & 43,4 & 60 & 56,6 & \multirow{2}{*}{0,671} \\
\hline & Femenino & 38 & 40,4 & 56 & 59,6 & \\
\hline \multirow{2}{*}{ Grupo etario } & Adolecente & 30 & 43,5 & 39 & 56,5 & \multirow{2}{*}{0,759} \\
\hline & Adulto medio & 54 & 41,2 & 77 & 58,8 & \\
\hline \multirow{3}{*}{ Estrato social } & Bajo & 6 & 30,0 & 14 & 70,0 & \multirow{3}{*}{$0,047^{*}$} \\
\hline & Medio & 64 & 48,1 & 69 & 51,9 & \\
\hline & Alto & 14 & 29,8 & 33 & 70,2 & \\
\hline \multirow{3}{*}{ Ciclo de formación } & Básico & 33 & 45,2 & 40 & 54,8 & \multirow{3}{*}{0,389} \\
\hline & Profesional & 34 & 37,0 & 58 & 63,0 & \\
\hline & Profundización & 17 & 48,6 & 18 & 51,4 & \\
\hline \multirow{2}{*}{ Programa } & Medicina & 42 & 42,0 & 58 & 58,0 & \multirow{2}{*}{1,000} \\
\hline & Otros & 42 & 42,0 & 58 & 58,0 & \\
\hline \multirow{2}{*}{ Ocupación } & Estudia & 77 & 41,2 & 110 & 58,8 & \multirow{2}{*}{0,371} \\
\hline & Estudia y trabaja & 7 & 53,8 & 6 & 46,2 & \\
\hline
\end{tabular}

*El estadístico es significativo en el 0,05.

Fuente: elaboración propia.

La frecuencia de ansiedad no presentó asociación estadística con la edad (Valor $p$ Prueba U de Mann-Whitney=0,509) ni el número de créditos matriculados (Valor $\mathrm{p}$ Prueba $U$ de Mann-Whitney= 0,652 ). Al desagregar el análisis según la gravedad de la ansiedad no se hallaron diferencia estadísticas en las con variables analizadas.

\section{Discusión}

La prevalencia de ansiedad en el actual grupo fue $58 \%-55 \%$ en grado leve y $3,0 \%$ moderado-. Este resultado resulta significativamente menor que el promedio reportado para Colombia en la última encuesta de salud mental, donde fue de $19,3 \%$ en toda la vida ( $16 \%$ en hombres y $21,8 \%$ en mujeres); $11,7 \%$ en los últimos 12 meses $(9,1 \%$ en hombres y $13,7 \%$ en mujeres); y $5,1 \%$ los últimos 30 días (3,7\% en hombres y 6,2\% en mujeres) (Ministerio de la Protección Social de Colombia, 2003).

Cabe aclarar que estos datos hacían parte del estudio que la Organización Mundial de la Salud (WHO, 2004) desarrolló entre 2001 y 2003 sobre salud mental, donde se encuestaron 60.463 adultos de los cuales 4544 eran colombianos, en quienes la prevalencia cercana al $20 \%$ evidenció que la ansiedad en el país es un importante problema de salud, y ocupa los primeros renglones en cuanto a motivos de consulta psicológica y psiquiátrica (Posada, Aguilar y Gómez, 2004; Bohórquez, 
2007; Ministerio de la Protección Social, 2003). Lo anterior implica que en estudiantes universitarios es un problema aún mayor, dada su prevalencia, casi del doble, frente al promedio nacional.

Estudios previos desarrollados en estudiantes universitarios han reportado prevalencias de ansiedad similares y diferentes a las del actual estudio así: Riveros, Hernández y Rivera (2007), en Lima,Perú, reportaron un 58\%; Balanza, Morales y Guerrero (2009) indicaron que el $55,7 \%$ de los encuestados sufrían algún tipo de trastorno de ansiedad; en la Universidad Industrial de Santander (UIS, 2004), en Bucaramanga, se reportó que de las consultas atendidas en el Departamento de Bienestar Universitario en 2004, el trastorno de ansiedad se presentó en un $9 \%$ de los estudiantes; y en la Universidad Pontificia Bolivariana (UPB, 2005) el resultado fue $17 \%$. Esto corrobora la necesidad de disponer de datos para cada grupo particular, dadas las divergencias que se pueden presentar según el grupo de estudio.

Específicamente en estudiantes de medicina, en Bogotá la prevalencia de ansiedad marcó un $60 \%$, $9 \%$ moderada y $1 \%$ grave (Bohórquez, 2007), mientras que en países como Perú, Brasil y otros se ha reportado que al menos $47 \%$ de los encuestados presenta un problema de salud mental; $19 \%$, ansiedad (Weiss, Warner, Lyketsos, Frank, Ganzini y Carter, 2001); 13,2\%, trastorno de ansiedad generalizada (Galli, Feijóo, Roig y Romero, 2002); y $35,4 \%$, síntomas ansiosos (Gómez y Carmeiro, 2005).

En el actual estudio, la prevalencia de ansiedad no presentó asociación con el sexo, la edad, el ciclo de formación, el programa académico ni la ocupación. Al evidenciar que esta enfermedad es un problema generalizado en todos los subgrupos analizados, solo se halló una distribución estadísticamente diferente según el estrato social, siendo menor su prevalencia en los estudiantes de clase media. En contraste, otros estudios han referido su asociación con el sexo, el semestre académico, condiciones socioeconómicas, entre otras, como se indica en las líneas siguientes.

Con base en el sexo, son múltiples los estudios que han referido que en población universitaria, las mujeres tienden a puntuar más alto que los hombres las preguntas relacionadas con ansiedad en diferentes pruebas de tamización (Agudelo, Casadiegos y Sánchez, 2008), y esto se corrobora con investigaciones que han hallado una mayor prevalencia de ansiedad en las mujeres (Riveros, Hernández y Rivera, 2007). Incluso algunos han referido un riesgo mayor a 2,0 de padecer ansiedad en las mujeres frente a los hombres (Balanza, Morales y Guerrero, 2009).

En cuanto al ciclo de formación, su asociación ha sido más referida en estudiantes de medicina que en otras carreras; se ha encontrado que los estudiantes de los primeros semestres de medicina tienen mayores niveles de estrés $y$, en consecuencia, mayor riesgo de desarrollar trastornos mentales. Estudios previos han reportado prevalencias de ansiedad de $26,4 \%$ en estudiantes del primer año, y de $8,9 \%$ en el sexto (Celis, Bustamante, Cabrera, Cabrera, Alarcón y Monge, 2001). Otros han encontrado mayor prevalencia de ansiedad en el ciclo básico que en las asignaturas clínicas (Bohórquez, 2007). Esto se puede explicar por las modalidades de evaluación y el hecho de realizar prácticas curriculares extrauniversitarias en centros de salud, lo que implica retos para articular clases en la universidad y en dichos centros; además, la realización de prácticas clínicas, siguiendo el turno del tutor, implica frecuentes cambios del ciclo vital/sueño, que se consideran un factor estresante (Balanza, et al. 2009; Brewer, 2002). En general, el mayor grado de estrés que presentan los estudiantes está ligado a su labor y a la exigencia académica, (Bohórquez, 2007), además de otros factores como un menor tiempo de sueño, un menor tiempo para recreación y cuidado personal, y una menor interacción social con amigos u otros grupos (Firth, 2001).

No obstante lo anterior, otros reportes coinciden con el del actual grupo de estudio, donde se observa que no existen diferencias significativas entre los estudiantes de inicio de carrera, mitad de carrera y salida a práctica (Agudelo et al., 2008).

Entre los principales factores académicos asociados con la ansiedad se han referido la insatisfacción con la carrera, dificultades en las relaciones 
con compañeros y docentes, y el estrés generado por las responsabilidades académicas (Riveros et al. 2007).

En general, la mayoría de estudios citan como principales factores asociados con la ansiedad en estudiantes aspectos de orden afectivo, económico, de ubicación de la residencia durante el curso académico (los alumnos que residen en el domicilio familiar presenta una menor frecuencia de ansiedad que los residentes en otros lugares), la necesidad de compaginar una actividad laboral de manera simultánea a la actividad académica, las cargas familiares, los factores asociados con la disminución del mantenimiento de la red de socialización y la percepción de un menor apoyo social (Riveros et al. 2007; Balanza et al. 2009; Roberts y Goldgin, 1999; Jou y Fukada, 2002).

La relevancia de estudiar la ansiedad radicó en que esta, aunada al estrés postraumático, el trastorno de angustia, la fobia social y la agorafobia sin pánico, constituyen los trastornos que generan mayor discapacidad, $y$, entre las condiciones neuropsiquiátricas, están entre las primeras causas de años de vida perdidos (Posada et al. 2006; WHO, 2001). Además, este problema afecta la salud mental, psicológica y social; compromete la atención, la capacidad de concentración y la destreza para la toma de decisiones; y genera rendimiento académico insatisfactorio y deserción escolar (Bohórquez, 2007; Riveros et al., 2007; Agudelo et al., 2008; Shapiro, Shapiro y Schwartz, 2000).

Varios estudios han encontrado que la identificación temprana de este trastorno no sólo minimiza la posibilidad de fracaso académico, sino que también reduciría en forma substancial otras conductas de riesgo para la salud, como el consumo de cigarrillo o de alcohol, o las conductas alimentarias desadaptativas (Agudelo et al., 2008; Campo, Díaz, Rueda y Barros, 2005).

Entre las principales limitaciones del estudio se encuentran las inherentes al tipo de estudio. En este sentido se debe precisar la presencia de sesgo temporal y el carácter exploratorio de las asociaciones estadísticas; no se evaluaron aspectos de orden socio-económico que podrían explicar la elevada prevalencia de ansiedad hallada, o deter- minar si esta obedece a una respuesta adaptativa más que patológica. Además, en la comparación de la prevalencia de ansiedad y sus factores asociados, algunos estudios de referencia emplearon escalas diferentes de tamización de la enfermedad, lo que puede generar algunas diferencias en las medidas de ocurrencia expuestas. En este sentido, se debe recabar que el test de Zung empleado está validado para la población colombiana y presentó excelente fiabilidad en el grupo estudiado.

\section{Conclusión}

Se halló una elevada prevalencia de ansiedad a partir de la aplicación de una escala validada para la población colombiana, y con excelente desarrollo psicométrico para la población de estudio; esto pone de manifiesto la necesidad de desarrollar estrategias educativas, identificar estudiantes de mayor riesgo e iniciar intervenciones en salud de manera oportuna.

\section{Referencias}

Acuña, J., Botto, A., y Jiménez, J. (2009). Psiquiatría para la atención primaria y el médico general. Depresión, ansiedad y somatización. Santiago de Chile: Mediterráneo.

Agudelo, D., Casadiegos, C., y Sánchez, D. (2008). Características de ansiedad y depresión en estudiantes universitarios. International Journal of Psychological Research, 1(1), 34-39.

Alba L. (2010). Salud de la adolescencia en Colombia: Bases para una medicina de prevención. Universitas Médica 51(1), 29-42.

Balanza, S., Morales, I., y Guerrero, J. (2009). Prevalencia de ansiedad y depresión en una población de estudiantes universitarios: Factores académicos y socio-familiares asociados. Cínica y Salud, 20(2), 177-187.

Bohórquez, A. (2007). Prevalencia de depresión y ansiedad según las escalas de Zung y evaluación de la asociación con el desempeño 
académico en los estudiantes de medicina de la Pontificia Universidad Javeriana. Bogotá: Pontificia Universidad Javeriana.

Brewer, T. (2002). Test-taking anxiety among nursing and general college students. Journal of Psychosocial Nursing and Mental Health, 40(11), 22-31.

Campo, A., Díaz, L., Rueda, G., y Barros, J. (2005). Validación de la escala de Zung para depresión en universitarias de Bucaramanga, Colombia. Revista Colombiana de Psiquiatría, 34(1), 54-62.

Celis, J., Bustamante, M., Cabrera, D., Cabrera, M., Alarcón, W., y Monge, E. (2001). Ansiedad y estrés académico en estudiantes de medicina humana del primer y sexto año. Anales de la Facultad de Medicina, 62(1), 25-30.

Firth J. (2001, enero). Medical student stress. Med Educ, 35(1), 6-7.

Galli, E., Feijóo, L., Roig, I., y Romero, S. (2002). Aplicación del "MINI" como orientación diagnostica psiquiátrica en estudiantes de medicina de la Universidad Peruana Cayetano Heredia: Informe preliminar epidemiológico. Rev. Med. Hered., 13(1), 19-25.

Gómez, F., y Carmeiro, M. (2005). Stress in medical students at the Federal University in Ceará, Brazil. Rev. bras. Edu. Méd, 29(2), 91-6.

Sarason, I., y Sarason, B. (1996). Psicopatología: Psicología anormal; El problema de la conducta inadaptada (7. ${ }^{\text {a }}$ ed). México: Prentice Hall.

Jou, Y., \& Fukada, H. (2002). Stress, health, and reciprocity and sufficiency of social support: The case of university students in Japan. The Journal of Social Psychology, 142(3), 353-370.

Kaplan, H., Sandock, B., y Sadock, V. (2009). Sinopsis de psiquiatría (10. ${ }^{a}$ ed.). Filadelfia, PA: Lippincott Williams \& Wilkins.

Ministerio de Salud y Protección Social. (2003). Informe descriptivo: Encuesta Nacional de
Salud Mental. Bogotá: Ministerio de Salud y Protección Social.

Ministerio de Salud y Protección Social. (2003). Un panorama nacional de la salud y enfermedad mental de la salud en Colombia: Informe preliminar; Estudio nacional de salud mental en Colombia. Recuperado el 23 de mayo de 2006 de http://www.abacolombia.org.co/bv/clinica/estudiosaludmentalcolombia.pdf

Posada, J., Aguilar, M., y Gómez, L. (2004). Prevalencia de los trastornos mentales y uso de servicios: Resultados preliminares del estudio nacional de salud mental; Colombia 2003. Revista Colombiana de Psiquiatría, 33(3), 241-262.

Posada, J., Buitrago, J., Medina, Y., y Rodríguez, M. (2006). Trastornos de ansiedad según distribución por edad, género, variaciones por regiones, edad de aparición, uso de servicios, estado civil y funcionamiento/discapacidad según el estudio nacional de salud mentalColombia. NOVA Publicación Científica, 4(6), 33-41.

Riveros, M., Hernández, H., y Rivera, J. (2007). Niveles de depresión y ansiedad en estudiantes universitarios de Lima Metropolitana. Revista de Investigación en Psicología, 10(1), 91-102.

Roberts, R., \& Goldgin, J. (1999). The effects of economic circumstances on British Students' mental and physical health. Journal of American College Health, 48(3), 103-110.

Shapiro, S., Shapiro, D., \& Schwartz, G. (2000). Stress management in medical education: A review of literature. Acad. Med., 75, 748-59.

Universidad Industrial de Santander (UIS), Departamento de Bienestar Universitario. (2005). Reporte de consultas atendidas según trastorno y/o diagnóstico establecido en el año 2004. Documento de trabajo inédito. Departamento de Bienestar Universitario, Universidad Industrial de Santander (UIS), Bucaramanga.

Universidad Pontificia Bolivariana (UPB), Departamento de Bienestar Universitario. (2005). Re- 
porte de consultas atendidas según trastorno y/o diagnóstico establecido en los meses de junio a septiembre del año 2005. Documento de trabajo inédito, Departamento de Bienestar Universitario, Universidad Pontificia Bolivariana, Bucaramanga.

Weiss, L., Warner, T., Lyketsos, C., Frank, E., Ganzini, L., \& Carter D. (2001). Perceptions of academic vulnerability associated with per- sona illness: A study of 1027 students at nine medical schools. Comprehensive Psychiatry, 42(1), 1-15.

World Health Organization (WHO). (2001). World health report: Mental health new understanding. New Hope: WHO.

World Health Organization (WHO). (2004). World mental survey consortium. JAMA, 291, 2581-90. 